\title{
Optimal Management of Acute Recurrent Laryngeal Nerve Injury During Thyroidectomy
}

\author{
Tova Fischer Isseroff • Michael Jay Pitman
}

Published online: 17 May 2013

(C) Springer Science+Business Media New York 2013

\begin{abstract}
Recurrent laryngeal nerve (RLN) injury is commonly encountered by thyroid surgeons and may carry with it great morbidity. Whether the injury is accidental or due to deliberate resection for oncologic soundness, the surgeon must be prepared to offer the best available treatment to patients, either at the time of injury or early in the recovery process. We review advances in treatment that allow optimal management of an acute RLN injury: ansa cervicalis-RLN reinnervation, in-office unsedated vocal fold injection augmentation, and electromyographic prognostication. The benefits and advantages of each procedure as well as relevant anatomy and techniques are delineated.
\end{abstract}

Keywords Recurrent laryngeal nerve - Ansa cervicalis . Laryngeal reinnervation - Thyroidectomy · Vocal fold paralysis · Dysphonia

\section{Introduction}

Recurrent laryngeal nerve (RLN) injury is a severe consequence of head and neck surgery. It causes dysphonia, dysphagia, and occasionally significant aspiration. In

\section{T. F. Isseroff}

Department of Otolaryngology-Head and Neck Surgery, The New York Eye and Ear Infirmary, 310 East 14th St., 6th Floor, New York, NY 10003, USA

e-mail: tfischer@nyee.edu

\section{J. Pitman ( $₫)$}

Department of Otolaryngology, The Voice and Swallowing Institute, The New York Eye and Ear Infirmary, 310 East

14th St., 6th Floor, New York, NY 10003, USA

e-mail:mpitman@nyee.edu addition to patient morbidity, injury also results in medicolegal challenges and significant loss of worker productivity at a high cost to both patients and society [1]. Those who operate in the region of the RLN are obligated to strive to protect the nerve and minimize patient morbidity by optimizing management when injury occurs.

This is especially true for thyroid surgeons, where intraoperative preservation of the RLN is a priority. Testament to this is the shear number of publications produced over many years on the topic [2••]. Consequently, at significant cost, new technology with indeterminate benefit has been developed and used to aid in localization and preservation of the RLN [3, 4]. Despite this, RLN injury is still common. During thyroidectomy, the incidence of permanent postoperative RLN paralysis is $0.3-3 \%$ and is as high as $2-30 \%$ in revision thyroid surgery $[2 \bullet \cdot]$.

Owing to the increasing rate of surgery for thyroid cancer, it is likely the incidence of iatrogenic RLN injury will be even higher in the coming years. The incidence of thyroid cancer in the USA has been rising since the mid$1990 \mathrm{~s}$, and has increased by $5.5 \%$ per year in males and $6.6 \%$ in females since 2004. This growth continues because of both improved detection methods and higher incidence rates in an expanding US population [5]. Approximately 60,220 new cases of thyroid cancer are expected to be diagnosed in the USA in 2013, with 3 in 4 cases occuring in women [6].

One of the areas of highest preoperative concern for thyroidectomy patients is experiencing a postoperative voice change [7]. Poor vocal outcome from an RLN injury is of real concern to physicians and a focal point of medicolegal action. Approximately $46 \%$ of thyroidectomy malpractice cases are related to RLN injury [8]. Over a 24-year period, an estimated $\$ 95$ million was paid out in indemnity payments because of thyroidectomy-related 
malpractice claims. The average payment was $\$ 437,852$ adjusted for inflation [9].

Considering the above facts, RLN injury will continue to occur with increasing frequency. In contrast to the extensive literature considering the prevention of RLN injury, few studies are devoted to the optimal the management of an acutely injured RLN. To best serve patients and minimize their morbidity, surgeons must be prepared to treat an acutely injured RLN whether it is identified in the operating room or in the early postoperative period. Herein we discuss the optimal management of an acute RLN injury identified either intraoperatively or postoperatively, using ansa cervicalis (ANSA)-RLN reinnervation, in-office vocal fold injection augmentation, and laryngeal electromyography (EMG).

\section{Intraoperative Recognition}

\section{Recurrent Laryngeal Nerve Reinnervation}

The RLN may be injured inadvertently or purposefully resected to allow oncologic resection of thyroid cancer. When discontinuity of the RLN is recognized intraoperatively, options exist: do nothing and rely on a secondary surgical procedure in the future such as a laryngoplasty, reanastomose the RLN, reconnect the RLN with a nerve graft, or reinnervate the RLN with an alternative nerve source.

Although relying on future surgery for vocal rehabilitation is an option, it is far from optimal, as the patient will suffer the morbidity related to the injury as well as a secondary surgical procedure. In contrast, successful reinnervation of the RLN will obviate the need for a second surgical procedure and demonstrate expertise in cases of inadvertent nerve injury. To decide on the best reparative option, the pathophysiology of RLN reinnervation must be understood as well as the advantages and disadvantages of the different techniques available.

The RLN is a mixed nerve with random positioning of both adductor and abductor axons [10]. When the axons of the nerve are transected, they regenerate in random fashion with some adductor axons innervating abductor muscles and vice versa [11]. This results in synkinetic reinnervation. In 2000, Crumley [12] detailed the clinical characteristics of synkinesis, describing four types of synkinesis to explain various laryngeal phenomena after recovery: type I-an immobile or poorly mobile vocal fold with unaffected voice; type II-spasmodic or twitching vocal folds; type III-hyperadducted vocal folds; type IVhyperabducted vocal folds with possible aspiration. Only type I was deemed "favorable." Owing to unfavorable synkinetic reinnervation, RLN-RLN reanastamosis or free nerve grafts can result in dysfunctional vocal folds, resulting in morbidity requiring intervention to correct the detrimental effects of synkinetic reinnervation [13, 14]. Overall, however, RLN-RLN or free graft anastomosis appears to result in favorable synkinetic reinnervation approximately $70-75 \%$ of the time, although the study series are small [14-17].

Considering this success rate, if both nerve endings are identified and a tension-free anastomosis can be performed, although suboptimal, RLN-RLN reanastomosis is a viable option. In the situation of an inadvertently transected RLN, this reanastomosis should be performed at minimum, as without it the patient will inevitably need further surgery for vocal rehabilitation [14]. If it appears the anastomosis will be under tension, a greater auricular nerve graft can be used. The results are the same as with RLN-RLN, but the graft is in a separate surgical field and results in anesthesia of the ear lobule and surrounding skin. Other options for reinnervation include the neuromuscular pedicle, nerve implantation, and ANSA, hypoglossal, or vagus nerve to RLN anastomosis. Neuromuscular pedicle and nerve implantation do not appear to be as successful as ANSARLN reinnervation, and harvesting all or part of the vagus or hypoglossal nerve may result in significant patient morbidity [18••, 19].

The ANSA is an ideal nerve for RLN reinnervation. There is no morbidity related to its sacrifice. During a thyroidectomy, it is close to the surgical field and is rarely injured unless an aggressive neck dissection is being performed. The ANSA is approximately $1 \mathrm{~cm}$ from the RLN, so the nerve length needed for this short transposition is minimal (Fig. 1). Because of this, a tension-free anastomosis is nearly always possible. The ANSA has a diameter similar to that of the RLN and can be used for both ipsilateral and contralateral anastamosis [12, 20, 21]. The

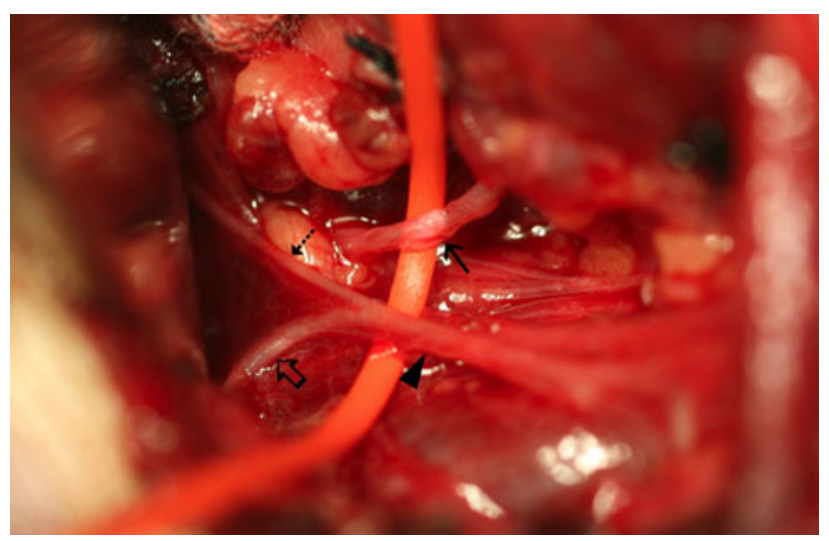

Fig. 1 Left recurrent laryngeal nerve (solid arrow) and superior root of the ansa cervicalis (arrowhead) with loop (hollow arrow) and common branch (dashed arrow). Note the proximity of the nerves after mobilization prior to transection 
ANSA also naturally fires during phonation, and there is increased recruitment with increasing vocal volume [22, 23]. Success of ANSA-RLN reinnervation is attributed to restoration of bulk and tone in glottic musculature resulting in medialization of the vocal fold and correction of arytenoid malposition and muscular atrophy. Owing to the innervation of the adductor and abductor muscles with one type of axon, synkinesis does not occur as with RLN-RLN anastomosis. Additionally, the anatomic structure of the larynx is preserved. This allows subsequent injection augmentation or type 1 laryngoplasty to be performed in the $1-2 \%$ of cases in which failure occurs. One limitation to ANSA-RLN reinnervation is that in elderly patients, reinnervation is not as robust, and they may have increased risk of failure [24•,25].

Although first described in 1924 by Frazier [26], ANSA-RLN reinnervation had been dormant until Crumley [27] published his original series in 1986. In 1991, he published an update of 20 cases, of which seven and five patients had "excellent" and "normal" voice outcomes, respectively [28]. The study included three patients with preoperative synkinesis, corrected following ANSA-RLN reinnervation. One patient required postoperative injection medialization to improve voice quality, and one patient with a history of multiple prior laryngeal surgical procedures and subglottic stenosis did not show any voice improvement. Vocal fold position, muscle mass, and tension were restored and no synkinesis was seen. A $95 \%$ success rate was reported for ANSA-RLN reinnervation.

In 2008, Lorenz et al. [25] studied a series of 46 patients who underwent delayed ANSA-RLN anastomosis with a median time to repair of 12 months. Thirty-seven of 38 patients who were followed up for a minimum of 3 months showed evidence of reinnervation and did not need a secondary procedure, a $97 \%$ success rate. All patients who underwent preoperative and postoperative Consensus Auditory-Perceptual Evaluation of Voice (CAPE-V) and laryngovideostroboscopy showed improvement in voice severity, roughness, breathiness, and strain as well as glottic closure, straightened vocal fold edge, and decreased supraglottic effort.

Wang et al. [18••] performed a case-control study looking at the long-term efficacy of delayed ANSA-RLN reinnervation following thyroid cancer surgery. In a large series, 237 Chinese patients underwent ANSA-RLN reinnervation 6-42 months after surgery. The patients were followed for a mean of 5 years and matched with 237 controls. On laryngovideostroboscopy, $92.4 \%$ of the patients had straight vocal fold edges, median or near-median vocal fold position, and symmetric and regular vocal fold vibration, with complete glottic closure during phonation. Significant improvement was demonstrated using the GRBAS scale. Maximum phonation time, jitter, shimmer, and noise to harmonic ratio were equivalent to those of controls. Reinnervation was confirmed on laryngeal EMG by the presence of significantly increased recruitment of motor unit action potentials in the thyroarytenoid muscle with phonation. Only four of 237 patients failed to restore adequate phonation. Two failures were due to technical reasons; one anastomosis was performed under significant tension and the other was avulsed during evacuation of a hematoma. Only two failures were due to inadequate reinnervation. Essentially, $98-99 \%$ of the ANSA-RLN reinnervations were successful.

Aynehchi et al. [21] systematically reviewed the outcomes from 14 case series of laryngeal reinnervation procedures for unilateral vocal fold paralysis between 1966 and 2009. Owing to the heterogeneity of the available literature and difficulty in analyzing techniques and outcomes, it is difficult to extrapolate direct data comparing different techniques. However, an improvement in acoustic, perceptive, EMG, and glottic gap analysis was seen with all reinnervation techniques. ANSA-RLN anastomosis was the commonest reinnervation technique, performed in $43.5 \%$ of procedures following thyroidectomy. It resulted in statistically significant better glottal closure than the other techniques, with a mean postsurgical time to reinnervation of 4.5 months.

It is clear that ANSA-RLN reinnervation works well when performed in a delayed fashion, with results similar to those of a type 1 laryngoplasty [24•]. Either can be performed for treatment of a chronic injury, but only ANSARLN reinnervation is available during surgery at the time of an acute injury. It is in the acute injury scenario when ANSA-RLN reinnervation is most powerful, especially in the face of a preoperative vocal fold paralysis or after RLN resection for cancer. The success of acute reinnervation is as good as that of delayed anastomosis, and the presence of malignancy does not affect the outcome of reinnervation.

Sanuki et al. [29] reported a series of 12 patients with thyroid cancer who experienced either nerve sacrifice or injury intraoperatively. Six patients had preoperative vocal fold paralysis and six underwent intraoperative sacrifice. Reconstruction was performed immediately using direct anastamosis $(n=1)$, free nerve graft $(n=9)$, or ANSARLN reinnervation $(n=2)$. Postoperative vocal perceptual analysis scores, aerodynamic function, and laryngovideostroboscopy findings were improved in all groups compared with values obtained before surgery from patients with preoperative vocal fold paralysis.

More detailed and extensive work on acute RLN reinnervation has been performed in Japan by Miyauchi et al. [20]. They retrospectively reviewed a series of patients who had undergone immediate reconstruction of the RLN after thyroid cancer resection. Thirty-four patients underwent reinnervation procedures, of which 19 were ANSARLN reinnervation [direct anastamosis $(n=5)$, free nerve 
graft $(n=8)$, vagus-RLN anastomosis $(n=2)$, and ANSA-RLN reinnervation $(n=19)$ ]. Recovery of voice began between 2 and 5 months postoperatively, with maximum improvement at 12 months. On laryngoscopy, patients had a minimal glottal gap, with good bulk and tension of the vocal folds. Maximum phonation time was not significantly different from that in the 34 normal controls. A most important insight from Miyauchi et al. was that once they began using ANSA-RLN reinnervation, it became their preferred method of reconstruction. This was due to consistent access to the nerve, the available length of the ANSA for a tension-free anastomosis, no morbidity related to the graft harvest, and the ease of the procedure performed primarily with surgical loupes. Once they had adopting ANSA-RLN reinnervation, they had a statistically significant increase in their anastomosis rate from 18 to $82 \%$, further proving the utility of this method.

In 2009, Miyauchi et al. [30] reviewed 88 cases of RLN reconstruction after nerve sacrifice during thyroid surgery. Sixty-five patients had reconstruction with ANSA-RLN anastomosis. Patients' voices began to improve within 3-5 months. Laryngoscopy revealed midline vocal folds with good tension and tone as well as a minimal glottal gap. Recovery of phonatory function was assessed quantitatively with the phonation efficiency index (PEI), a ratio of maximum phonation time to vital capacity. Eighty-seven percent of patients had a PEI similar to that of normal subjects at 1 year. The validity of the PEI, which Miyauchi et al. constructed, is unclear. Of note, $47 \%$ of anastomoses were performed intralaryngeally to the distal end of the RLN. This is critical because RLN injury or cancer invasion often occurs close to the cricothyroid joint. This leaves only a small section of exposed distal nerve, making performing an anastomosis technically difficult. In these cases, incision of the inferior constrictor with removal of the inferior horn of the thyroid cartilage exposes extra length of usually undisturbed RLN. This extra length of intralaryngeal nerve allows a tension-free anastomosis to be performed despite a distal nerve injury.

\section{Operative Technique and Anatomy}

\section{Anatomy}

To successfully perform an ANSA-RLN reinnervation, a surgeon must be mentally prepared, know the relevant anatomy, and understand the surgical technique. The ANSA is formed from two nerve roots that join inferiorly to form a loop. The superior root, or the descendens hypoglossi, receives branches of communication from $\mathrm{C} 1$ and C2 and comes off cranial nerve XII, whereas the inferior root arises from the junction of the cervical rami, usually C2-C4 [31]. The superior root leaves cranial nerve XII just inferior to the origin of the occipital artery and descends obliquely along the carotid artery. The inferior root passes posterolaterally to the internal jugular vein 57-81\% of the time, otherwise passing medially. The two branches join as a loop deep to the superior belly of the omohyoid, at the point where the muscle crosses the great vessels. The loop is in this location $64 \%$ of the time, although it may rest anywhere between the occipital artery and $4 \mathrm{~cm}$ above the sternum. Following formation of the loop, the nerve branches innervate the sternohyoid and sternothyroid. The common ANSA branch is approximately $4.3 \mathrm{~cm}$ long and $0.9 \mathrm{~mm}$ wide, making it a good anatomic fit in length and diameter for anastamosis to the RLN [32]. In the past, the nerve branch to the sternothyroid or common branch was used with good surgical outcome [12]. However, the ascending fibers or descending fibers of the superior root are slightly easier to mobilize and are commonly used for ANSA-RLN reinnervation as well. An elegant study of the ANSA using Sudan black to stain the axons noted a greater number of motor axons traveling cranially versus caudally in the superior root of the ANSA [31]. Considering this, the best nerve to use for reinnervation may be the ascending aspect of the superior root.

\section{ANSA-RLN Reinnervation Procedure}

Variations of the procedure have been described; however, the most important factor is exposure of the relevant nerve endings and achieving a tension-free anastomosis. To identify the ANSA, the superior belly of the omohyoid can be found crossing beneath the sternocleidomastoid muscle. The omohyoid is then dissected from surrounding fascia and retracted superiorly where it overlies the internal jugular vein. The loop of the ANSA is generally just beneath the muscle. The common branch to the strap muscles is then identified and dissected distally. A branch similar in thickness to the ANSA should be chosen, transected, and mobilized toward the RLN. Alternatively, the superior root of the ANSA can be dissected cranially. The root is then transected far enough cranially so the ascending aspect of the ANSA can be mobilized and sutured to the RLN without tension. On occasion, the RLN is injured or sacrificed at the cricothyroid joint; as such, a distal stump is not readily available for anastamosis. In this case, the inferior constrictor muscle can be divided and the inferior horn of the thyroid cartilage removed to identify an additional $0.5-1 \mathrm{~cm}$ of intralaryngeal RLN [18••, 30]. When the ipsilateral ANSA has been resected or its viability is in question, a contralateral ANSA anastomosis can be performed by routing the nerves anterior to the thyroid cartilage [33••]. Nerve edges are trimmed sharply and epineurium is exposed and gently peeled back to allow separation and identification of fascicles. Three to four 9.0 
nylon sutures are used to approximate the nerve ends. Care should be taken to suture only the epineurium and protect the fascicles from injury. The sutures should approximate the nerve endings so they are not strangulated or do not overlap. Performing the anastomosis with either surgical loupes or an operating microscope does not appear to affect vocal outcome [20].

\section{Temporary Injection Augmentation}

As it takes 3-5 months for the reinnervation to significantly improve the voice, a temporary bridging injection augmentation of the paralyzed vocal fold should to be performed [20]. This can be conducted in the operating room if the contralateral RLN was not placed at risk during the surgery and is known to be functioning normally. If the status of the contralateral nerve is unknown, the patient should be awoken and adequate motion of the corresponding vocal fold confirmed. The injection is then performed which the patient unsedated, under topical anesthesia at any time when that the patient is mentally and physically ready. Typically, the injection is performed 1-2 weeks following surgery, but can be performed as early as the first postoperative day (see later for injection details).

The combination of ANSA-RLN reinnervation and an early vocal fold injection results in a patient having only 1-2 weeks of morbidity from their vocal fold paralysis, as long as the reinnervation is successful, as is expected $97-99 \%$ of the time. This algorithm for treatment relegates one of the most feared complications of thyroid surgery to a minimally morbid treatable circumstance.

\section{Postoperative Recognition}

\section{Injection Augmentation}

If a patient has symptomatic vocal fold paralysis recognized in the early postoperative period, the "watching and waiting" approach is no longer the preferred method of treatment. Injection augmentation of the vocal fold can be performed in the office soon after thyroid surgery, under topical anesthesia with minimal patient morbidity or discomfort and with a low complication rate [34, 35•]. As evidence of its utility, the rate of in-office injections has eclipsed that of those performed in the operating room [35']. Additional benefit is realized by the decreased cost of an injection performed in the office compared with the operating room [36].

There are many options for temporary injectable materials [37•]. The choice is usually based on duration of the material's persistence, as well as the surgeon's familiarity and success with it. The authors prefer micronized AlloDerm as it causes minimal inflammation and is very forgiving. Its drawback is that it must be reconstituted prior to use. There are also multiple approaches to performing the injection: peroral, transcutaneous, or transnasal [38•]. The authors prefer the thyrohyoid approach as described by Achkar et al. [39॰] and Amin [40] with a needle bending modification. The benefit of most injections lasts 3-4 months and allows the patient good phonatory function while reinnervation occurs. As the injected material resorbs, vocal fold bulk, position, and phonatory function will be maintained by the newly innervating axons, whether arising from a reinnervated RLN or an injured but intact RLN.

The benefits of early vocal fold injection go beyond that of just "positioning" the vocal fold while reinnervation occurs. It appears that early vocal fold injection decreases patients' need for a secondary laryngoplasty. Friedman et al. [41•] retrospectively reviewed 35 patients with postsurgical or idiopathic vocal fold paralysis into whom hyaluronic acid gel has been injected within 1 year of injury. Twenty of thirty-two patients $(62.5 \%)$ with injection medialization performed less than 6 months following injury maintained adequate voice outcomes, whereas all three patients with injection later than 6 months after injury needed further reconstruction. Yung et al. [42••] retrospectively reviewed 54 patients with vocal fold paralysis and also found that patients who underwent temporary injection medialization were less likely to undergo permanent medialization laryngoplasty $(26 \%)$ as compared with patients who were conservatively managed $(65 \%)$.

The decreased need for intervention after early vocal fold injection is likely secondary to more rapid and robust reinnervation. Experiments involving reinnervation of the facial nerve demonstrate that periodic stimulation of an animal's whiskers will increase the rate and function of facial nerve regeneration [43•, 44]. This concept can be applied to RLN reinnervation. If the vocal folds are brought into apposition with an injection augmentation, the paralyzed vocal fold will receive vibrotactile and afferent nerve stimulation during phonation that it otherwise would not have had if it were lateralized and injection augmentation had not been performed. This stimulation may parallel that of whisker stimulation, hence increasing the rate and robustness of RLN reinnervation. In this vein, voice therapy may be beneficial as an adjunctive treatment, providing patients exercise and mandated vocal fold stimulation be performed throughout the day.

\section{Electromyographic Prognostication}

Electromyographic prognostication is an important adjunct in the care of patients in whom RLN paralysis is 
recognized postoperatively. Two to 6 months after injury, EMG helps predict which patients may regain vocal fold function and which are unlikely to recover. Temporary augmentation should be offered to those awaiting recovery, whereas early laryngoplasty for permanent medialization would benefit those with minimal chance of return of vocal fold function. Thus, EMG can be used to guide treatment, limit unnecessary temporary procedures, and speed the time to full rehabilitation.

Munin et al. [45] have investigated the role of EMG in evaluating synkinesis and prognosticating recovery. In their original study performed in 2003 , they retrospectively evaluated 31 patients with vocal fold paralysis for whom EMG was performed less than 6 months after injury. An excellent prognosis was predicted by EMG findings of normal motor unit recruitment and configuration with a full or slightly decreased interference pattern and no fibrillation potentials. A poor prognosis was determined by diminished motor unit recruitment, repetitive motor unit firing, and a decreased interference pattern with spontaneous activity. A fair prognosis showed moderately decreased motor unit recruitment, diminished interference pattern, and normal or polyphasic motor unit potentials. The positive predictive value for recovery of vocal fold motion was $66.7 \%$, as four of six patients prognosticated as excellent showed recovery. Only in five of 25 patients with a poor prognosis was there resolution, giving a negative predictive value of $80 \%$.

In 2010 the same group proposed an EMG protocol for determination of adductor synkinesis to combine with their previous criteria. Forty-six patents with vocal fold paralysis and available EMG data were retrospectively reviewed. Synkinesis was determined by the ratio between motor unit potentials in the thyroarytenoid muscle during adductor and abductor tasks. Synkinesis was defined as a ratio of 0.65 or greater. For patients with good motor unit recruitment who prognostically had potential for excellent recovery using their original EMG criteria, the presence of synkinesis downgraded them to a poor prognosis. This addition of synkinesis to the protocol improved the negative predictive value of EMG from 53 to $100 \%$, whereas the positive predictive value was $76 \%[46 \bullet]$.

Smith et al. [47] further improved the positive predictive value of EMG by adding quantitative analysis of wave amplitude and turns. Adding this information to the previous criteria improved the positive predictive value to $100 \%$, whereas the negative predictive value dropped slightly to $89.5 \%$, with seven of eight correct predictions.

\section{Conclusions}

RLN injury after thyroid surgery can cause significant patient morbidity. Surgeons treating this injury should be aware of the optimal treatment following injury. If it is recognized intraoperatively, using the most current techniques of ANSA-RLN reinnervation and in-office vocal fold injection augmentation will restore the voice within 1-2 weeks of surgery. If RLN injury is recognized postoperatively, early vocal fold injection will decrease the length of disability and the need for a secondary operation, whereas EMG will shorten the waiting time to permanent rehabilitation and minimize the unnecessary use of further temporary procedures. Together, these management techniques decrease patient morbidity, demonstrate surgical and medical expertise, and decrease costs to society associated with lost worker productivity, extra surgical procedures, and medicolegal costs.

Disclosure Tova Fischer Isseroff and Michael Jay Pitman both declare no conflict of interest.

\section{References}

Papers of particular interest, published recently, have been highlighted as:

- Of importance

•• Of major importance

1. Spector BC, Netterville JL, Billante C, Clary J, Reinisch L, Smith TL. Quality-of-life assessment in patients with unilateral vocal cord paralysis. Otolaryngol Head Neck Surg. 2001;125(3):176-82.

2. • Hayward NJ, Grodski S, Yeung M, Johnson WR, Serpell J. Recurrent laryngeal nerve injury in thyroid surgery: a review. ANZ J Surg 2013;83(1-2):15-21. This is an excellent and thorough review of the literature regarding $R L N$ injury in thyroid surgery, with a focus on the identification of high-risk patients, the role of intraoperative identification and dissection of the nerve, and the role of intraoperative neuromonitoring and optimal preoperative nerve assessment.

3. Dionigi G, Bacuzzi A, Boni L, Rausei S, Rovera F, Dionigi R. Visualization versus neuromonitoring of recurrent laryngeal nerves during thyroidectomy: what about the costs? World J Surg. 2012;36(4):748-54.

4. Higgins TS, Gupta R, Ketcham AS, Sataloff RT, Wadsworth JT, Sinacori JT. Recurrent laryngeal nerve monitoring versus identification alone on post-thyroidectomy true vocal fold palsy: a meta-analysis. Laryngoscope. 2011;121(5):1009-17.

5. Agency for Healthcare Research and Quality. Statistical brief \#86. Healthcare Cost and Utilization Project (HCUP). 2010. http:// www.hcup-us.ahrq.gov/reports/statbriefs/sb86.jsp. Accessed 3 Aug 2012.

6. American Cancer Society. Cancer facts and figures 2013. 2013. http://www.cancer.org/acs/groups/content/@epidemiology surveilance/documents/document/acspc-036845.pdf. Accessed 5 May 2013.

7. Abdul-Sater L, Henry M, Majdan A, Mijovic T, Franklin JH, Brandt MG, et al. What are thyroidectomy patients really concerned about? Otolaryngol Head Neck Surg. 2011;144(5): 685-90.

8. Abadin SS, Kaplan EL, Angelos P. Malpractice litigation after thyroid surgery: the role of recurrent laryngeal nerve injuries, 1989-2009. Surgery. 2010;148(4):718-22; discussion 722-3. 
9. Singer MC, Iverson KC, Terris DJ. Thyroidectomy-related malpractice claims. Otolaryngol Head Neck Surg. 2012;146(3):358-61.

10. Gacek RR, Malmgren LT, Lyon MJ. Localization of adductor and abductor motor nerve fibers to the larynx. Ann Otol Rhinol Laryngol. 1977;86(6 Pt 1):771-6.

11. Flint PW, Downs DH, Coltrera MD. Laryngeal synkinesis following reinnervation in the rat. Neuroanatomic and physiologic study using retrograde fluorescent tracers and electromyography. Ann Otol Rhinol Laryngol. 1991;100(10):797-806.

12. Crumley RL. Laryngeal synkinesis revisited. Ann Otol Rhinol Laryngol. 2000;109(4):365-71.

13. Woo P, Mangaro M. Aberrant recurrent laryngeal nerve reinnervation as a cause of stridor and laryngospasm. Ann Otol Rhinol Laryngol. 2004;113(10):805-8.

14. Chou FF, Su CY, Jeng SF, Hsu KL, Lu KY. Neurorrhaphy of the recurrent laryngeal nerve. J Am Coll Surg. 2003;197(1):52-7.

15. Ezaki H, Ushio H, Harada Y, Takeichi N. Recurrent laryngeal nerve anastomosis following thyroid surgery. World J Surg. 1982;6(3):342-6

16. Yumoto E, Sanuki T, Kumai Y. Immediate recurrent laryngeal nerve reconstruction and vocal outcome. Laryngoscope. 2006; 116(9):1657-61.

17. Green DC, Ward PH. The management of the divided recurrent laryngeal nerve. Laryngoscope. 1990;100(7):779-82.

18. • Wang W, Chen D, Chen S, Li D, Li M, Xia S, et al. Laryngeal reinnervation using ansa cervicalis for thyroid surgery-related unilateral vocal fold paralysis: a long-term outcome analysis of 237 cases. PLoS One 2011;6(4):e19128. This is the largest series of ANSA-RLN reinnervations, including 237 patients and a success rate of $98-99 \%$.

19. Zheng H, Zhou S, Chen S, Li Z, Cuan Y. An experimental comparison of different kinds of laryngeal muscle reinnervation. Otolaryngol Head Neck Surg. 1998;119(5):540-7.

20. Miyauchi A, Matsusaka K, Kihara M, Matsuzuka F, Hirai K, Yokozawa $\mathrm{T}$, et al. The role of ansa-to-recurrent-laryngeal nerve anastomosis in operations for thyroid cancer. Eur J Surg. 1998; 164(12):927-33.

21. Aynehchi BB, McCoul ED, Sundaram K. Systematic review of laryngeal reinnervation techniques. Otolaryngol Head Neck Surg. 2010;143(6):749-59.

22. Zheng H, Li Z, Zhou S. Electromyographic study of human extrinsic laryngeal muscles. Zhonghua Er Bi Yan Hou Ke Za Zhi. 1995;30(2):108-10.

23. Faaborg-Andersen K, Vennard W. Electromyography of extrinsic laryngeal muscles during phonation of different vowels. Ann Otol Rhinol Laryngol. 1964;73:248-54.

24. - Paniello RC, Edgar JD, Kallogjeri D, Piccirillo JF. Medialization versus reinnervation for unilateral vocal fold paralysis: a multicenter randomized clinical trial. Laryngoscope 2011; 121(10):2172-2179. This is the only randomized clinical trial comparing the gold standard of medialization versus reinnervation for the treatment of unilateral vocal fold paralysis. The results of ANSA-RLN reinnervation were similar to those of medialization laryngoplasty.

25. Lorenz RR, Esclamado RM, Teker AM, Strome M, Scharpf J, Hicks D, et al. Ansa cervicalis-to-recurrent laryngeal nerve anastomosis for unilateral vocal fold paralysis: experience of a single institution. Ann Otol Rhinol Laryngol. 2008;117(1):40-5.

26. Frazier $\mathrm{CH}$. The treatment of paralysis of the recurrent laryngeal nerve by nerve anastomosis. Ann Surg. 1924;79(2):161-71.

27. Crumley RL, Izdebski K. Voice quality following laryngeal reinnervation by ansa hypoglossi transfer. Laryngoscope. 1986; 96(6):611-6.

28. Crumley RL. Update: ansa cervicalis to recurrent laryngeal nerve anastomosis for unilateral laryngeal paralysis. Laryngoscope. 1991;101(4 Pt 1):384-7; discussion 388.
29. Sanuki T, Yumoto E, Minoda R, Kodama N. The role of immediate recurrent laryngeal nerve reconstruction for thyroid cancer surgery. J Oncol. 2010;2010:846235.

30. Miyauchi A, Inoue H, Tomoda C, Fukushima M, Kihara M, Higashiyama $\mathrm{T}$, et al. Improvement in phonation after reconstruction of the recurrent laryngeal nerve in patients with thyroid cancer invading the nerve. Surgery. 2009;146(6):1056-62.

31. Banneheka S. Anatomy of the ansa cervicalis: nerve fiber analysis. Anat Sci Int. 2008;83(2):61-7.

32. Chhetri DK, Berke GS. Ansa cervicalis nerve: review of the topographic anatomy and morphology. Laryngoscope. 1997; 107(10):1366-72.

33. • Wang W, Chen S, Chen D, Xia S, Qiu X, Liu Y, et al. Contralateral ansa cervicalis-to-recurrent laryngeal nerve anastomosis for unilateral vocal fold paralysis: a long-term outcome analysis of 56 cases. Laryngoscope 2011;121(5):1027-1034. This provides an excellent description of the technique and evaluates the effectiveness of contralateral ANSA-RLN reinnervation.

34. Young VN, Smith LJ, Sulica L, Krishna P, Rosen CA. Patient tolerance of awake, in-office laryngeal procedures: a multiinstitutional perspective. Laryngoscope. 2012;122(2):315-21.

35. - Sulica L, Rosen CA, Postma GN, Simpson B, Amin M, Courey $\mathrm{M}$, et al. Current practice in injection augmentation of the vocal folds: indications, treatment principles, techniques, and complications. Laryngoscope 2010;120(2):319-325. This is a multiinstitutional retrospective review of patients undergoing vocal fold injection augmentation at seven university medical centers. The findings show the adoption of awake office injection as the preferred method, with low complication rates equivalent to those of injection under general anesthesia.

36. Bove MJ, Jabbour N, Krishna P, Flaherty K, Saul M, Wunar R, et al. Operating room versus office-based injection laryngoplasty: a comparative analysis of reimbursement. Laryngoscope. 2007;117(2):226-30.

37. - Mallur PS, Rosen CA. Vocal fold injection: review of indications, techniques, and materials for augmentation. Clin Exp Otorhinolaryngol 2010;3(4):177-182. An excellent review of available vocal fold injection materials and techniques.

38. - Mallur PS, Rosen CA. Office-based laryngeal injections. Otolaryngol Clin North Am 2013;46(1):85-100. This is an excellent illustrated review of the technical aspects of performing in-office vocal fold injection via multiple routes.

39. • Achkar J, Song P, Andrus J, Franco R Jr. Double-bend needle modification for transthyrohyoid vocal fold injection. Laryngoscope 2012;122(4):865-867. This describes an important modification to simplify access to the vocal fold via the thyrohyoid approach during in-office injection augmentation.

40. Amin MR. Thyrohyoid approach for vocal fold augmentation. Ann Otol Rhinol Laryngol. 2006;115(9):699-702.

41. • Friedman AD, Burns JA, Heaton JT, Zeitels SM. Early versus late injection medialization for unilateral vocal cord paralysis. Laryngoscope 2010;120(10):2042-2046. This is the first article showing the long-term benefit of early vocal fold injection, reducing the need for a secondary medialization laryngoplasty.

42. •• Yung KC, Likhterov I, Courey MS. Effect of temporary vocal fold injection medialization on the rate of permanent medialization laryngoplasty in unilateral vocal fold paralysis patients. Laryngoscope 2011;121(10):2191-2194. This is the primary article showing the long-term benefit of vocal fold injection with a temporary material. Patients who underwent a vocal fold injection were significantly less likely to need a permanent medialization laryngoplasty, at a rate of $26 \%$ compared with $66 \%$ of those who were treated conservatively.

43. - Lindsay RW, Heaton JT, Edwards C, Smitson C, Vakharia K, Hadlock TA. Daily facial stimulation to improve recovery after facial nerve repair in rats. Arch Facial Plast Surg 
2010;12(3):180-185. This is a rat study on facial nerve reinnervation showing improved reinnervation with sensory stimulation. This is a likely reason for the long-term benefits of early vocal fold injection.

44. Angelov DN, Ceynowa M, Guntinas-Lichius O, Streppel M, Grosheva M, Kiryakova SI, et al. Mechanical stimulation of paralyzed vibrissal muscles following facial nerve injury in adult rat promotes full recovery of whisking. Neurobiol Dis. 2007; 26(1):229-42.

45. Munin MC, Rosen CA, Zullo T. Utility of laryngeal electromyography in predicting recovery after vocal fold paralysis. Arch Phys Med Rehabil. 2003;84(8):1150-3.
46. - Statham MM, Rosen CA, Smith LJ, Munin MC. Electromyographic laryngeal synkinesis alters prognosis in vocal fold paralysis. Laryngoscope 2010;120(2):285-290. These authors have established criteria resulting in a high predictive value for electromyography.

47. Smith LJ, Rosen CA, Niyonkuru C, Munin MC. Quantitative electromyography improves prediction in vocal fold paralysis. Laryngoscope. 2012;122(4):854-9. 